\begin{tabular}{|l|c|c|c|r|}
\hline $\begin{array}{l}\text { Cuadernos de Investigación Geográfica } \\
\text { Geographical Research Letters }\end{array}$ & 2017 & N $^{\circ} 43(1)$ & pp. 189-208 & $\begin{array}{r}\text { ISSN 0211-6820 } \\
\text { eISSN 1697-9540 }\end{array}$ \\
\hline
\end{tabular}

\title{
BENEFICIOS DE LOS ACOLCHADOS DE PAJA Y PODA COMO PRÁCTICAS PARA LA GESTIÓN FORESTAL DE MONTES MEDITERRÁNEOS
}

\author{
P. HUESO-GONZÁLEZ*, J.F. MARTÍNEZ-MURILLO, \\ J.D. RUIZ SINOGA
}

\begin{abstract}
Departamento de Geografía, Universidad de Málaga, Instituto de Geomorfología y Suelos (IGSUMA).
\end{abstract} Andalucía Tech. Campus de Teatinos s/n, 29071 Málaga, España.

RESUMEN. En la mayoría de las propuestas forestales para ambientes semiáridos, la respuesta positiva de la recolonización vegetal se ve limitada por las condiciones climáticas y la escasez de agua y nutrientes presentes en el suelo. Es por ello que, en estos ambientes, se hace necesaria la búsqueda de métodos alternativos que, con bajo coste económico, garanticen el éxito de cualquier actuación forestal. Este estudio trata de analizar el efecto de dos tipos de acolchados orgánicos sobre la viabilidad de una reforestación bajo condiciones forestales semiáridas. Para el diseño experimental se ha establecido un set de 6 parcelas experimentales: (i) dos parcelas reforestadas y enmendadas con un mulch de paja (10 $\mathrm{Mg} \mathrm{ha}^{-1}$ ) (SM); (ii) dos parcelas reforestadas y enmendadas con un mulch de restos de poda (Pinus halepensis Mill.) (10 $\mathrm{Mg} \mathrm{ha}^{-1}$ ) (PM); $y$ (iii) dos parcelas reforestadas sobre suelo desnudo, conjunto control $(C)$. Todas las parcelas se han reforestado con el mismo número de plantas y patrón espacial de arbustos mediterráneos. Los resultados muestran que, 30 meses después de la reforestación, no se observan diferencias significativas en el contenido de carbono orgánico del suelo entre los suelos con acolchados orgánicos de paja o poda y el control. Sin embargo, sí se han observado diferencias en la cantidad y disponibilidad de agua útil para la vegetación. Esto se interpreta como una reducción del estrés hídrico post-transplante, que permite amortiguar la estacionalidad mediterránea, generando un efecto positivo para la supervivencia y crecimiento de las plantas reforestadas. De cara a la gestión forestal para ambientes semiáridos, podemos concluir que el tratamiento del suelo con acolchados orgánicos, paja o poda, se traduce en un aumento del éxito de los programas de reforestación. 


\section{Benefits of straw and pine mulch as forest management practices in Mediterranean forests}

ABSTRACT. In semiarid conditions, re-vegetation programs are associated with a high level of sapling mortality. For the proper management of Mediterranean forest environments, alternative low cost afforestation methods that ensure the survival of seedlings and have minimal environmental impact are needed. One way to improve soil conditions is to apply organic amendments to the soil. This study assessed the effects of two types of organic mulching on the afforestation success of a degraded Mediterranean semiarid area. The soil treatments tested were: i) afforestation in soil amended with straw mulch (10 $\left.\mathrm{Mg} \mathrm{ha}^{-1}\right)$; ii) afforestation in soil amended with mulch containing chipped branches of Aleppo Pine (Pinus halepensis Mill.) (10 $\mathrm{Mg} \mathrm{ha}^{-1}$ ); iii) control plots with no amendments were used and considered as afforested in natural conditions. The afforestation plan was performed following the same species and pattern of plantation in each plot. We hypothesized that in the context of dry-Mediterranean climatic conditions the use of organic amendments would enhance plant establishment and ensure successful afforestation. The results showed that afforestation success increased when soils were amended with straw and pine tree mulch. The amendments had no effect on soil organic carbon. However, both treatments showed an increase in the average soil water content respect to the control conditions. Thus, the available water content in soils amended with mulch was higher than in the control during most of the duration of the experiment, and, consequently, there was less water stress following afforestation. This was well related with the increment in sapling survival rates founded in straw and pine mulch treatments.

Palabras clave: reforestación, acolchados orgánicos, condiciones semiáridas, agua útil, suelos mediterráneos.

Key words: afforestation, organic mulching, semiarid conditions, available water for plants, Mediterranean soils.

Recibido: 11 de noviembre de 2016

Aceptado: 21 de diciembre de 2016

* Correspondencia: Paloma Hueso-González, Departamento de Geografía, Universidad de Málaga. Instituto de Geomorfología y Suelos (IGSUMA). Andalucía Tech. Campus de Teatinos s/n, 29071 Málaga, España. E-mail: phueso@uma.es

\section{Introducción}

Los diferentes usos del territorio que caracterizan la región mediterránea (agrícolas y ganaderos, campos abandonados y vegetación natural), durante la historia reciente, están principalmente relacionados con factores físicos, técnicos o razones socioeconómicas (Cerdà, 2001; Martínez-Murillo et al., 2016b). El impacto humano sobre el 
paisaje es, por tanto, cada vez más perjudicial debido a la expansión de la agricultura extensiva, el sobrepastoreo, los incendios o la deforestación (Casals et al., 2000). Todo ello afecta negativamente a las propiedades de los suelos mediterráneos y favorece los procesos de erosión y degradación de los mismos (Hueso-González, 2014).

Actualmente, la degradación del suelo afecta a más de 52 millones de hectáreas de tierra en los países de la Unión Europea (COM, 2002). Este problema es particularmente grave en las zonas semiáridas mediterráneas, donde los efectos de las actividades antrópicas mencionadas se suman a los problemas causados por los períodos prolongados de sequía y lluvias intensas e irregulares (Thornes, 1985). Bajo estas condiciones climáticas, la dinámica del carbono orgánico del suelo (SOC) ha demostrado ser muy sensible con aquellos factores relacionados con el cambio climático, así como con el tipo y grado de recubrimiento vegetal (Shachak et al., 1998; Casals et al., 2000; Maestre et al., 2003; Eaton et al., 2008; Novara et al., 2011).

En los ambientes forestales, el patrón espacial y temporal de la vegetación dominante tendrá una clara influencia sobre las pérdidas de suelo por erosión hídrica, pues influye de dos maneras: (i) interfiriendo en la energía asociada a los procesos erosivos (Thornes, 1990; Cerdà, 2001); y (ii) modificando las propiedades físico-químicas de los suelos que la albergan (Thornes, 1990; Castillo et al., 1997; Muñoz-Rojas et al., 2016c). Así, durante la estación seca mediterránea, la falta de agua en la matriz del suelo se traduce en una reducción drástica de los inputs de SOC y, por tanto, a largo plazo, en la pérdida de fertilidad de los mismos (Van Wesemael y Veer, 1992; Fons y Vallejo, 1997; Martínez-Murillo et al., 2016a; Muñoz-Rojas et al., 2016a). Por ello se considera como una práctica común la recuperación de la cubierta vegetal como una estrategia efectiva para mitigar, y aún revertir, los efectos de la desertificación (Nykvist, 1983; Tongway y Ludwing, 1996; Vallejo et al., 2000; Le Houérou, 2010).

En ambientes semiáridos, los proyectos de revegetación están siendo considerados por las distintas administraciones con responsabilidad en gestión ambiental como una de las prioridades en los programas de restauración y lucha contra el Cambio Climático (Gómez, 1999; Rojo, 2000; Ministerio de Medio Ambiente, 2002a, 2002b). Atendiendo a la Ley de Montes, y a los efectos de la misma, se incluyen los siguientes términos: (i) repoblación como introducción de especies forestales en un terreno mediante siembra o plantación, puede ser forestación o reforestación; (ii) forestación como repoblación, mediante siembra o plantación, de un terreno que era agrícola o estaba dedicado a otros usos no forestales; (iii) reforestación como reintroducción de especies forestales, mediante siembra o plantación, en terrenos que estuvieron poblados forestalmente hasta épocas recientes, pero que quedaron rasos a causa de talas, incendios, vendavales, plagas o enfermedades (Ministerio de Medio Ambiente, 2002a, 2002b). Sin embargo, en las propuestas forestales en ambientes semiáridos, la respuesta positiva de la recolonización vegetal se podría ver limitada por la escasez de agua y nutrientes del suelo (Lal, 1997; Castillo et al., 2001; Brevik, 2009; García-Orenes et al., 2012).

Bajo condiciones mediterráneas, la búsqueda de métodos alternativos que, con bajo coste económico, garanticen el éxito de cualquier actuación forestal es, por tanto, necesaria (Eldridge et al., 2012; Benigno et al., 2013). En este sentido numerosos 
estudios ya han evaluado el efecto del uso de enmiendas orgánicas sobre la fertilidad de suelos agrícolas (Guerrero et al., 2001; Ojeda et al., 2003; Ros et al., 2006; Jordán et al., 2010, 2011; Jiménez et al., 2013; Tejada y González, 2013); suelos erosionados (Cohen-Fernández y Naeth, 2013; Prats et al., 2013; Donn et al., 2014; Hosseini Bai et al., 2014; Cerdà et al., 2016; Prosdocimi et al., 2016) o suelos mineros (Eldridge et al., 2012; Benigno et al., 2013; Muñoz-Rojas et al., 2016b). Sin embargo, ninguno de estos estudios ha podido demostrar que en condiciones forestales mediterráneas exista una relación directa entre la adición del residuo orgánico y el éxito en la actuación forestal diseñada.

El objetivo de este estudio es analizar el efecto de las enmiendas orgánicas sobre la viabilidad de una reforestación bajo condiciones forestales semiáridas. Concretamente, se pretende evaluar los efectos de los acolchados de paja o poda como nuevas herramientas para la gestión forestal.

\section{Materiales y métodos}

\section{1. Área experimental}

El área experimental "El Pinarillo" se encuentra ubicada a 470 m s.n.m., en la parte alta de un abanico aluvial formado por conglomerados calcáreos y dentro del Parque Natural Sierra Tejeda, Almijara y Alhama (sureste de España). El clima es seco-mediterráneo (temperatura media de $18^{\circ} \mathrm{C}$, precipitación media $589 \mathrm{~mm}$ año ${ }^{-1}$ ). La vegetación dominante consiste en un bosque de pino abierto acompañado del típico matorral mediterráneo. Las parcelas experimentales objeto de este estudio se encuentran ubicadas en una zona agrícola abandonada en los años 50 del siglo XX y recolonizada, de forma natural, por matorral y herbáceas.

Los suelos son de tipo Leptosoles líticos y eútricos con textura franco arenosa (arena $=60 \%, \operatorname{limo}=32 \%$, arcilla $=8 \%$ ) y presentan un elevado contenido en gravas (total de gravas $=56 \%$; gravas $>\mathrm{f} 10 \mathrm{~mm}=31 \%$; grava $\mathrm{f} 2 \mathrm{~mm}=10 \%$; grava $\mathrm{f} 1 \mathrm{~mm}=15 \%$ ). El perfil edáfico dominante es tipo $\mathrm{ACk} / \mathrm{Bw}$, resultado de una elevada fracturación en la roca madre y con contenido de carbono orgánico medio-bajo (LUCDEME, 1986). Las propiedades y características de suelos se encuentran recogidas en la Tabla 1.

\subsection{Parcelas}

Para el diseño experimental se construyen 6 parcelas experimentales simultáneas en el tiempo. Se trata de parcelas experimentales cerradas de $2 \mathrm{~m}$ de ancho y $12 \mathrm{~m}$ de longitud, $24 \mathrm{~m}^{2}$ de superficie total, que se han orientado de forma paralela a la línea de máxima pendiente. Así, la pendiente registrada para las 6 parcelas se corresponde con un $7,5 \%$ y la orientación es $\mathrm{N} 170^{\circ}$.

Para garantizar la uniformidad de las parcelas, fue eliminada la cubierta vegetal que de forma natural existía en el área experimental. Esto se hizo con el fin de evitar interferencias en los resultados. Finalmente, los restos vegetales procedentes del desbroce de la vegetación fueron retirados. 
Tabla 1. Propiedades del suelo bajo condiciones naturales en el área experimental El Pinarillo. CIC: capacidad de intercambio catiónico; CT: Carbono total; NT: Nitrógeno total; C/N: ratio carbono/nitrógeno; EC: Conductividad eléctrica; SOC: contenido de carbono orgánico; SWC: Contenido de agua en el suelo.

\begin{tabular}{|c|c|c|}
\hline Precipitación anual & $\left(\mathrm{mm}\right.$ año $\left.{ }^{-1}\right)$ & 589,0 \\
\hline Régimen climático & & Seco \\
\hline Especies dominantes & & $\begin{array}{c}\text { Chamaerops humilis, Cistus } \\
\text { albidus, Rosmarinus offici- } \\
\text { nalis }\end{array}$ \\
\hline \multicolumn{3}{|l|}{ Propiedades del suelo } \\
\hline Gravas & $(\%)$ & 56,0 \\
\hline Arena & $(\%)$ & 60,0 \\
\hline Limo & $(\%)$ & 32,0 \\
\hline Arcilla & $(\%)$ & 8,0 \\
\hline $\begin{array}{r}\text { Capacidad de campo } \\
\text { SWC }\end{array}$ & $(\%)$ & 25,2 \\
\hline $\begin{array}{r}\text { Punto de marchitez } \\
\text { SWC }\end{array}$ & $(\%)$ & 6,4 \\
\hline Agua disponible & $(\%)$ & 18,8 \\
\hline Estabilidad estructural & $(\%)$ & 51,0 \\
\hline CIC & meq $100 \mathrm{~g}^{-1}$ & 120,4 \\
\hline CT & $(\%)$ & 12,5 \\
\hline NT & $(\%)$ & 0,2 \\
\hline $\mathrm{C} / \mathrm{N}$ & & 74,0 \\
\hline SOC & $(\%)$ & 1,9 \\
\hline $\mathrm{pH}$ & & 8,0 \\
\hline $\mathrm{EC}$ & $\left(\mu \mathrm{S} \mathrm{cm}^{-1}\right)$ & 501,0 \\
\hline
\end{tabular}

\subsection{Enmiendas}

Posteriormente, en mayo de 2011, se añaden al suelo, en 4 de las 6 parcelas, dos tipos diferentes de acolchados orgánicos. Los acolchados seleccionados fueron: (i) acolchado de restos de paja (SM); y (ii) acolchado de astillas de pino carrasco (Pinus halepensis Mill.) (PM).

En este experimento, cada tratamiento ha sido replicado en dos parcelas experimentales. El patrón de repetición ha sido al azar. Por último, las dos enmiendas seleccionadas se han aplicado a la misma dosis (10 $\left.\mathrm{Mg} \mathrm{ha}^{-1}\right)$.

\subsection{Reforestación}

En noviembre de 2011, seis meses después de la aplicación de las enmiendas, las 6 parcelas han sido reforestadas con el mismo número de plantas y patrón espacial de arbustos mediterráneos. Así, la vegetación se ha reforestado utilizando un patrón al tres 
bolillo con 0,5 m de distancia entre plantones. Durante el proceso de reforestación, el suelo se volteó hasta los $25 \mathrm{~cm}$ de profundidad. Las especies vegetales utilizadas han sido Lavadula stoechas Lam., L. dentatae L., L. multifida L., R. officinalis L. y T. capitatus L. En este estudio la condición control (C) la conforman 2 parcelas experimentales que fueron reforestadas pero no enmendadas.

\subsection{Muestreo, análisis de las propiedades del suelo y mediciones}

El suelo de las parcelas reforestadas fue muestreado en: (i) primavera del año 1 (6 meses después de la repoblación forestal); (ii) otoño del año 1 (12 meses después de la repoblación forestal); (iii) primavera del año 2 (18 meses después de la repoblación forestal); y (iv) otoño del año 2 (24 meses después de la repoblación forestal). La estrategia de muestreo para cada parcela consistía en la toma de una colección de 4 muestras de suelo alterado que fueron tomadas en superficie $(0-10 \mathrm{~cm}$ de profundidad). Las propiedades del suelo analizadas han sido: (i) SOC, que se determina mediante la oxidación con dicromato potásico y posterior titulación, vía método de Walkley-Black (FAO, 2006); (ii) capacidad de retención de agua en el suelo (WHC), que se determina usando cajas de arena ( $\mathrm{pF} 2$,0) y membrana de Richards ( $\mathrm{pF} 4$ 4) (Richards, 1947; Stakman et al., 1969; Martínez-Fernández et al., 1995). Por último, con el fin de evaluar el estado hidrológico del suelo a lo largo del año hidrológico y su relación potencial con la capacidad de agua disponible para las plantas (AWC), se ha medido el punto de marchitez (WP) y la capacidad de campo (FC) (Caldwell, 1976); (iii) humedad en continuo (SWC), con intervalo de10 minutos. Para ello se instalan en el área experimental 12 sondas de humedad tipo HOBO S-SMx-M005. Concretamente, se han emplazado 2 sondas por parcela, a una profundidad en el perfil de 5 y $10 \mathrm{~cm}$. La elección de las profundidades para las sondas se justifica por el limitado desarrollo del sistema radicular que presenta la vegetación reforestada en sus estadios iniciales (Hueso-González et al., 2015).

\subsection{Seguimiento y monitoreo de la vegetación}

La estrategia diseñada para el seguimiento espacio-temporal de la vegetación reforestada es la siguiente: con independencia del manejo y una vez reforestadas las parcelas experimentales, en el mes de mayo de 2012, coincidiendo con el fin de la primera primavera, antes del inicio de la sequía estival, los plantones plantados en cada parcela experimental se supervisaron y se procedió al conteo de marras. A partir de ese momento los plantones se han revisado dos veces al año durante el período 2012-2014. Concretamente, la frecuencia ha sido: (i) 6 meses después de la reforestación (primavera del año 1); (ii) 12 meses después de la reforestación; (iii) 20 meses después de la reforestación; (iv) 30 meses después de la reforestación. Esta frecuencia de monitoreo se seleccionó coincidiendo con el comienzo y fin de las estaciones secas y húmedas mediterráneas (Fig. 1). El conteo de los individuos se ha realizado in situ. Durante el conteo se ha anotado el estado fenológico de la planta (vivo o muerto) y la longitud del brote apical así como el diámetro mayor de la copa. Esta metodología ha sido seleccionada atendiendo a los criterios previamente establecidos por Castro et al. (2002) y Gómez-Aparicio et al. (2004). 


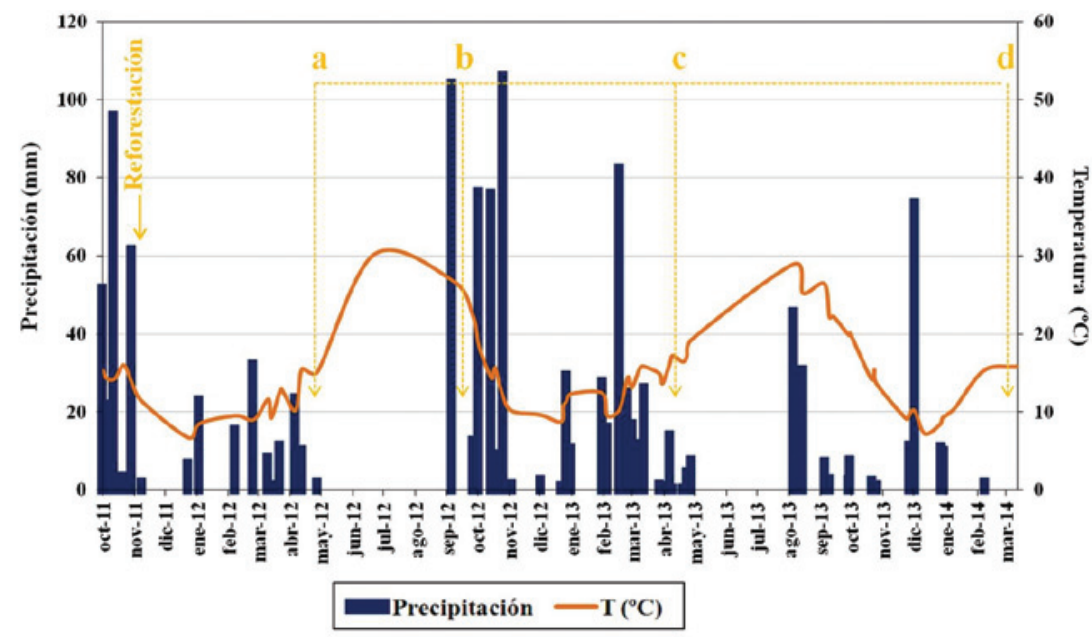

Figura 1. Variabilidad temporal de las precipitaciones y temperatura para el periodo octubre 2011 a marzo 2014. a: monitoreo de la vegetación 6 meses después de la reforestación; b: monitoreo de la vegetación 12 meses después de la reforestación; c: monitoreo de la vegetación 20 meses después de la reforestación; d: monitoreo de la vegetación 30 meses después de la reforestación. * indica las diferentes campañas de monitoreo de la vegetación.

\subsection{Tratamiento estadístico}

Las diferencias en las propiedades del suelo, SOC y SWC, se han medido utilizando la prueba de análisis de varianza para una vía (ANOVA de una vía). Las diferencias entre tratamientos se han determinado mediante el test de Tukey o Games-Howell. El límite de significancia seleccionado para los análisis es de $P<0,050$. La normalidad y homogeneidad de varianzas se verifica por las pruebas de Kolmogorov-Smirnov y Levene, respectivamente.

Para los datos de vegetación, se ha utilizado un análisis de modelos lineales generalizados (GLM, Bólker et al., 2009). Este análisis nos ha permitido controlar el efecto de parámetros fijos, como son las enmiendas o las especies, y los efectos aleatorios generados al replicar las parcelas. Además, para identificar diferencias significativas en el crecimiento $(\mathrm{P}<0,050)$ se ha aplicado el test de Tukey.

Todos los análisis se han llevado a cabo usando el software estadístico SPSS (versión 21) para Windows (IBM Corp. Released, 2012).

\section{Resultados y discusión}

\subsection{Tasas de supervivencia de la reforestación bajo condiciones de suelo desnudo}

En las regiones semiáridas mediterráneas, con una marcada sequía estival, el estrés post-plantación podría ser la causa principal de la mortalidad inicial de los plantones (Grossnickle, 2000; Bochet et al., 2004, 2007). Nuestros resultados, en la misma línea 
de los anteriores, ponen de manifiesto para condiciones de suelo desnudo, $\mathrm{C}$, que debido al régimen de precipitaciones, con lluvias escasas y torrenciales y un prolongado tiempo entre evento y evento, el establecimiento de la cubierta vegetal se ha visto dificultado (Fig. 1 y Tabla 2). Así, los porcentajes de marras encontrados, 30 meses después de la reforestación, alcanzaron valores de $62,5 \%, 26,0 \%, 41,9 \%, 25,0 \%$ y 43,7\% para las especies L. dentatae L., L. multifida L., Lavadula stoechas Lam., R. officinalis L. y T. capitatus $L$., respectivamente (Tabla 2 ).

Tabla 2. Tasas de supervivencia (\%) durante el periodo 2011-2014. C: suelos reforestados y no enmendados; PM: Acolchado de restos de poda de pino (Pinus halepensis Mill.); SM: acolchado de paja; $L d$ : Lavandula dentatae; Lm: Lavandula multifida; $L s$ : Lavandula stoechas; $R o$ :

Rosmarinus officinalis; $T c$ : Thymus capitatus.

\begin{tabular}{|c|c|c|c|c|}
\hline & \multirow[b]{2}{*}{ meses } & \multicolumn{3}{|c|}{ Supervivencia (\%) } \\
\hline & & C & SM & PM \\
\hline \multicolumn{5}{|c|}{$\mathrm{Ld}$} \\
\hline & 6 & 62,5 & 87,5 & 99,0 \\
\hline & 12 & 50,0 & 87,5 & 87,5 \\
\hline & 20 & 37,5 & 87,5 & 87,5 \\
\hline & 30 & 37,5 & 87,5 & 87,5 \\
\hline \multicolumn{5}{|c|}{$\mathrm{Lm}$} \\
\hline & 6 & 91,7 & 100,0 & 100,0 \\
\hline & 12 & 83,3 & 95,8 & 100,0 \\
\hline & 20 & 83,3 & 95,8 & 100,0 \\
\hline & 30 & 75,0 & 95,8 & 100,0 \\
\hline \multicolumn{5}{|l|}{ Ls } \\
\hline & 6 & 58,1 & 83,3 & 100,0 \\
\hline & 12 & 58,1 & 83,3 & 100,0 \\
\hline & 20 & 58,1 & 83,3 & 100,0 \\
\hline & 30 & 58,1 & 83,3 & 100,0 \\
\hline \multicolumn{5}{|c|}{ Ro } \\
\hline & 6 & 75,0 & 100,0 & 95,8 \\
\hline & 12 & 75,0 & 100,0 & 95,8 \\
\hline & 20 & 75,0 & 100,0 & 95,8 \\
\hline & 30 & 75,0 & 100,0 & 95,8 \\
\hline \multicolumn{5}{|l|}{ Tc } \\
\hline & 6 & 56,3 & 100,0 & 100,0 \\
\hline & 12 & 56,3 & 93,8 & 100,0 \\
\hline & 20 & 56,3 & 93,8 & 100,0 \\
\hline & 30 & 56,3 & 93,8 & 100,0 \\
\hline
\end{tabular}


Las bajas tasas de supervivencia encontradas para el conjunto control (C) se justifican por el escaso desarrollo radicular que presentan los plantones en los primeros año post-transplante. Esto imposibilitaría el acceso al agua contenida en las capas más profundas del perfil edáfico, siendo especialmente significativo durante la primera estación seca mediterránea (Maestre y Cortina, 2002; Maestre et al., 2002; Bochet et al., 2007).

\subsection{Efecto de los acolchados sobre las tasas de supervivencia}

Según lo establecido por Hasse y Rose (1993), en regiones semiáridas, la preparación del suelo constituye una práctica común que podría contribuir a reducir el estrés post-plantación de los plantones. En este sentido, varios estudios han demostrado que la adición de enmiendas orgánicas al suelo mejora la calidad de los mismos, lo que se debería interpretar como un efecto positivo para el desarrollo de la cubierta vegetal (Woods et al., 2012; Benigno et al., 2013; Hoseini Bai et al., 2014).

Para la prueba de GLM, nuestros resultados muestran diferencias para la supervivencia entre especies y entre los diferentes tratamientos del suelo aplicados (Tabla 3; $P=0,00$ y $P=0,00$, respectivamente). Concretamente, cuando los suelos se trataban con SM o PM, se observa un efecto positivo para las tasas de supervivencia respecto a los suelos no enmendados (C) (Tabla 4; $P=0,00$ y $P=0,00$, respectivamente). En la misma línea, Breton et al. (2016) han encontrado diferencias para el establecimiento de las plantas tras la adición de un mulch de astillas de madera. Estos autores, describen un aumento en las tasas de supervivencia de más del 50\% para las especies Buxus sempervirens, Quercus pubescens y Pinus nigra, tras la adición del acolchado orgánico. En este estudio, la Tabla 2 muestra que, con independencia del mulch aplicado (SM o PM), las especies Lavandula dentatae y Thymus capitatus, conseguían incrementar las tasas de supervivencia en más del $40 \%$ respecto a los suelos no enmendados. Para el resto de especies, también se ha medido un incremento en la supervivencia, aunque éste ha sido ligeramente inferior al anteriormente descrito (Tabla 2). Específicamente, especies como la Lavandula stoechas, Lavandula multifida y Rosmarinus officinalis habían aumentado en un 20-25\% la supervivencia respecto al control.

Tabla 3. Medida de la vegetación: modelos mixtos de efecto generalizado (GLM) para la supervivencia y el crecimiento, diámetro y altura, 30 meses después de la reforestación.

\begin{tabular}{lcc}
\cline { 2 - 3 } & $\mathrm{X}^{2}$ & $P$-valor \\
\hline Tratamiento & & \\
\hline Supervivencia & 43,94 & 0,00 \\
Altura & 61,38 & 0,00 \\
Diámetro & 64,66 & 0,00 \\
\hline Especie & & \\
\cline { 1 - 1 } Supervivencia & 13,73 & 0,01 \\
\cline { 1 - 1 } Altura & 61,38 & 0,00 \\
\cline { 1 - 1 } Diámetro & 07,91 & 0,00 \\
\hline
\end{tabular}


Tabla 4. Prueba Post-hoc de comparación múltiple para las medias observadas (prueba de Tukey, $P<0,05)$. SM: acolchado de paja; PM: acolchado de astillas de pino carrasco (Pinus halepensis Mill.); $C$ : suelos reforestados y no enmendados.* representa diferencias significativas con control.

\begin{tabular}{lcc}
\hline$P$-valor en relación a $C$ & SM & PM \\
\hline Supervivencia & $0,00^{*}$ & $0,00^{*}$ \\
Crecimiento en altura & $0,05^{*}$ & $0,00^{*}$ \\
Crecimiento en diámetro & $0,01^{*}$ & $0,04^{*}$ \\
\hline
\end{tabular}

\subsection{Efecto de los acolchados sobre la forma arquitectónica de los plantones}

$\mathrm{Si}$ atendemos a la forma arquitectónica de los plantones, se han encontrado marcadas diferencias en función de la especie utilizada y el tratamiento añadido (Tabla 3, $P<0,050)$. Por tanto, se hacen manifiestas diferencias significativas en la arquitectura de la planta, diámetro o altura, independientemente de la especie utilizada y tras la adición de los acolchados al suelo: SM (Tabla $4 ; P=0,05$ y $P=0,01$, respectivamente); y PM (Tabla $4 ; P=0,00$ y $P=0,04$, respectivamente).

Concretamente, en relación al control, los tratamientos SM y PM habían conseguido un aumento en altura para las especies Lavandula sp. y Thymus capitatus (Figs. 2, 3, 4 y $5 ; P<0,05)$. Si atendemos ahora al diámetro máximo de la copa, también se observa un incremento en el tamaño de la copa para las especies Lavandula stoechas y Lavandula multifida (Figs. 2 y 4; $P<0,05$ ). En relación a la especie Thymus capitatus, solo los suelos enmendados con el acolchado de paja (SM) registraron mayor crecimiento de la copa en comparación con los suelos no enmendados (Fig. $5 ; P<0,05$ ).

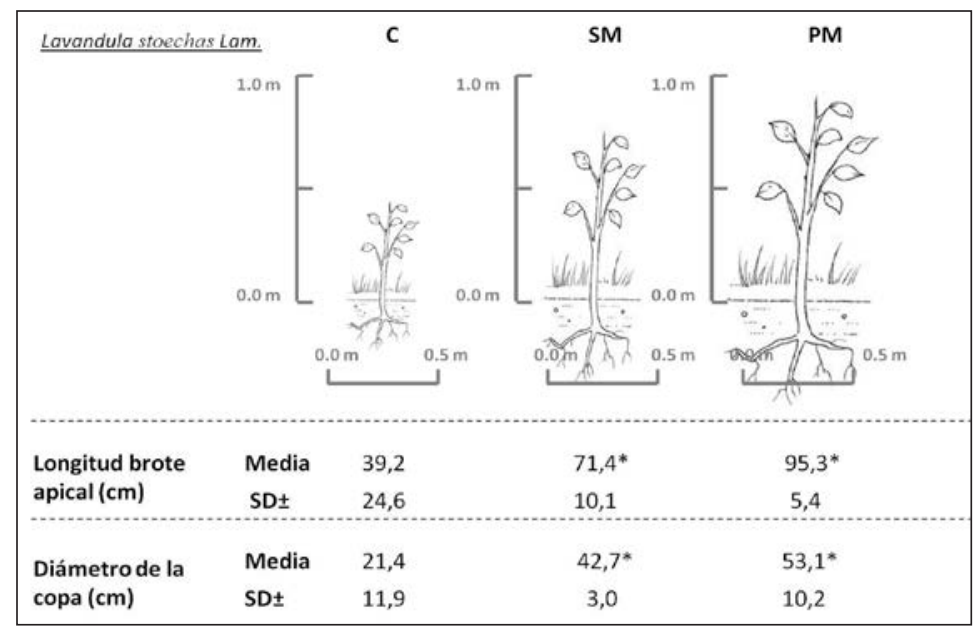

Figura 2. Lavandula stoechas Lam.: Crecimiento en altura de la vegetación (cm) y diámetro máximo de la copa $(\mathrm{cm}) 30$ meses después de la reforestación. C: suelos reforestados y no enmendados; PM: acolchado de restos de poda de pino (Pinus halepensis Mill.); SM: acolchado de paja. * indica diferencias significativas respecto al control $(C)(P<0,050)$. 


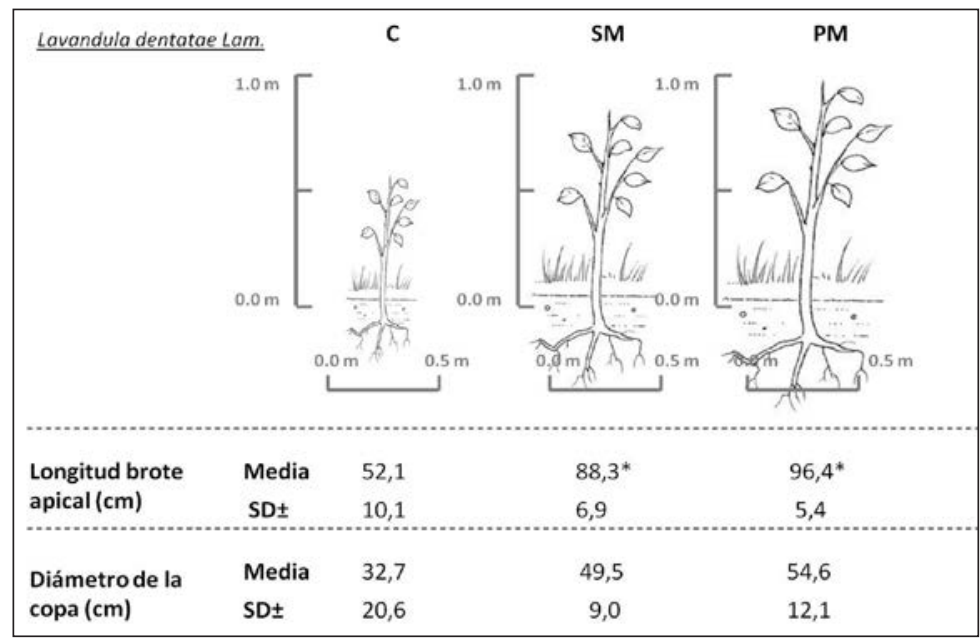

Figura 3. Lavandula dentatae Lam.: Crecimiento en altura de la vegetación (cm) y diámetro máximo de la copa 30 meses después de la reforestación. C: suelos reforestados y no enmendados; PM: acolchado de restos de poda de pino (Pinus halepensis Mill.); SM: acolchado de paja. * indica diferencias significativas respecto al control $(C)(P<0,050)$.

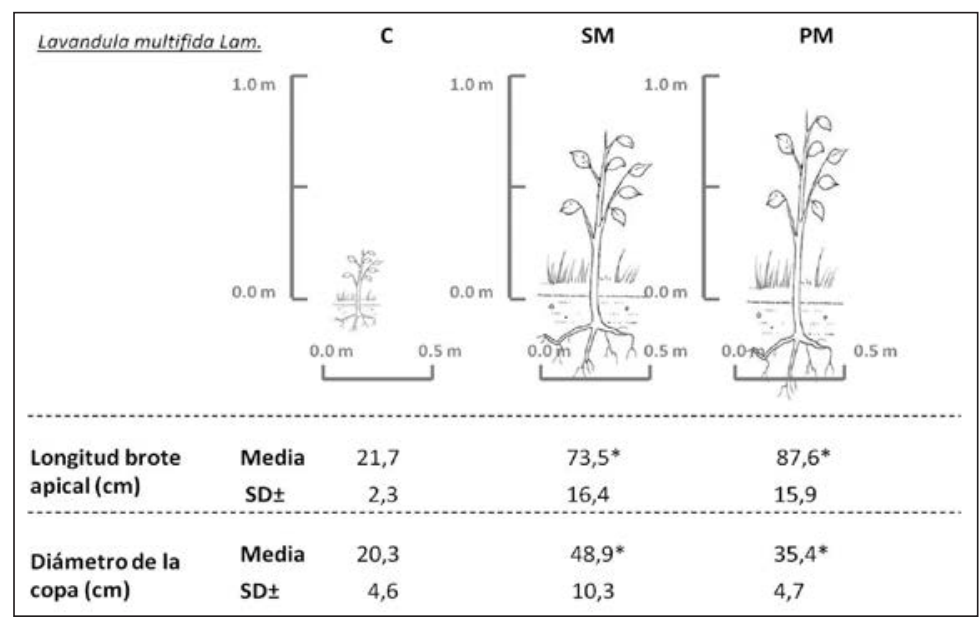

Figura 4. Lavandula multifida Lam.: Crecimiento en altura de la vegetación (cm) y diámetro máximo de la copa 30 meses después de la reforestación. C: suelos reforestados y no enmendados; PM: acolchado de restos de poda de pino (Pinus halepensis Mill.); SM: acolchado de paja. * indica diferencias significativas respecto al control $(C)(P<0,050)$.

En líneas generales, estos resultados se interpretan como que los acolchados de paja o poda no solo son capaces de reducir el porcentaje de marras frente a las reforestaciones ejecutadas bajo suelo desnudo sino que, además, consiguen que la vegetación sea capaz de establecerse y desarrollarse mejor, ya sea en diámetro, altura o ambos. 


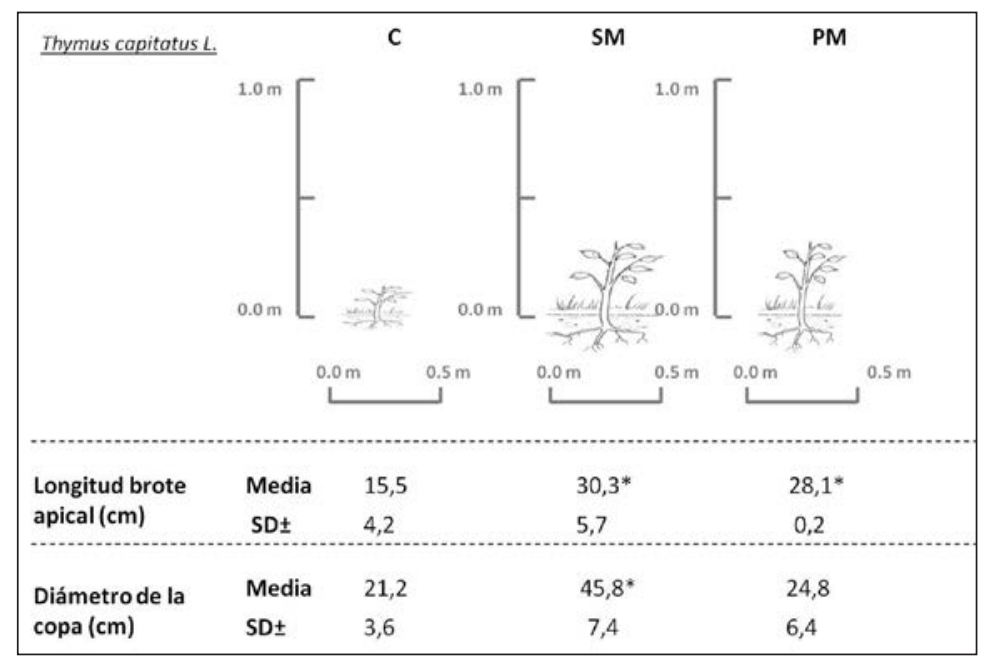

Figura 5. Thymus capitatus L.: Crecimiento en altura de la vegetación ( $\mathrm{cm}$ ) y diámetro máximo de la copa 30 meses después de la reforestación. C: suelos reforestados y no enmendados; PM: acolchado de restos de poda de pino (Pinus halepensis Mill.); SM: acolchado de paja. * indica diferencias significativas respecto al control $(C)(P<0,050)$.

\subsection{Efecto de los acolchados sobre el SOC}

En este estudio, los cambios en el SOC se han medido con el fin de determinar si estos permitirían justificar las diferencias descritas en el establecimiento y crecimiento de los plantones. Bajo condiciones semiáridas y en base a estudios previos, esto se justifica debido a que el SOC constituye un factor limitante durante el establecimiento de la vegetación (Almendros et al., 2010; Hueso-González et al., 2014).

Basándonos en otras investigaciones, se espera una disminución del porcentaje de marras cuando los suelos se modifican con algún aporte extra de materia orgánica por el incremento de SOC (Jordán et al., 2010, 2011; Chaudhuri et al., 2015; Shazana et al., 2013; Srinivasarao et al., 2013). De hecho, se ha demostrado para suelos agrícolas que la incorporación de residuos de cultivos, abonos orgánicos, lodos de depuradora o de estiércoles de diversos orígenes animales, genera un aumento de la materia orgánica y estaría directamente relacionado con un posible incremento en la producción de los cultivos (Montgomery, 2007; Ferreras et al., 2006; Franco-Otero et al., 2011; González-Ubierna et al., 2012).

Nuestros resultados para suelos forestales muestran que 30 meses después de la adición al suelo de las enmiendas SM y PM, no se observan diferencias significativas en los porcentajes de SOC respecto al control (Fig. 6; $P \geq 0,05$ ). En la misma línea están los resultados de González-Ubierna et al. (2012). La ausencia de diferencias en el SOC podría justificarse por las lentas tasas de mineralización de las enmiendas añadidas bajo condiciones semiáridas. En base a otros estudios, esto podría deberse a tres razones fundamentalmente: (i) la ausencia de compostaje previo en las enmiendas seleccionadas, que supondría un incremento significativo del tiempo necesario para que se produzcan los procesos de 
descomposición de la materia orgánica bajo climas mediterráneos (García-Gómez et al., 2005); (ii) el alto contenido de lignina y la celulosa presente en los tratamientos SM y PM, lo que aumentaría el tiempo necesario para la mineralización del SOC contenido en las mismas (Duryea et al., 1999; Jensen, 2009); y (iii) las dosis aplicadas, consideradas como medias-altas según otros autores (10 $\mathrm{Mg} \mathrm{ha}^{-1}$ ) (Jordán et al., 2010; Young et al., 2015). En este sentido, Jordán et al. (2010) han demostrado, para un área cultivada al suroeste de España, que las tasas de mineralización se aceleran cuando las modificaciones orgánicas se aplican a bajas dosis (3-5 $\mathrm{Mg} \mathrm{ha}^{-1}$ ) y se ralentizan a dosis altas (10-30 Mg ha-1). En la misma línea, Hueso-González et al. (2014) demuestran para enmiendas que no han sufrido compostaje previo, que serían necesarios más de 24 meses para observar cambios en los valores del SOC bajo condiciones semiáridas mediterráneas.

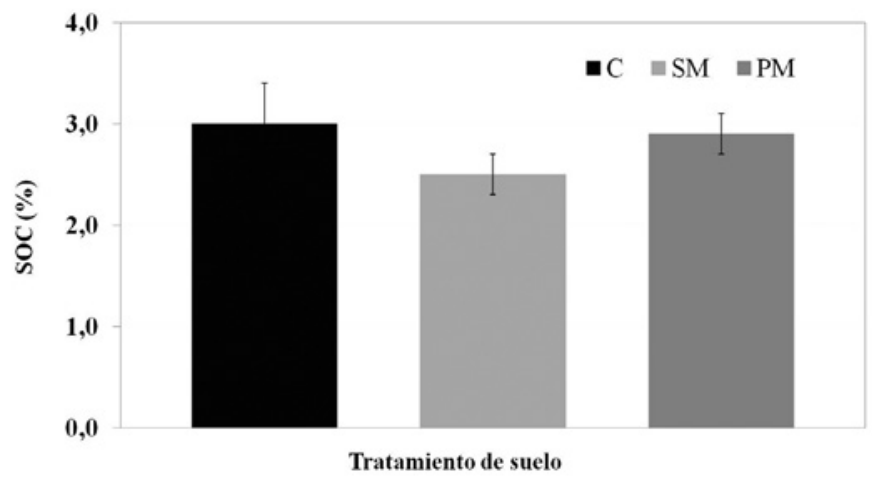

Figura 6. Carbono Orgánico del Suelo (SOC) medido 24 meses después de la reforestación. C: suelos reforestados y no enmendados; SM: acolchado de paja; PM: acolchado de astillas de pino carrasco (Pinus halepensis Mill.); * indica diferencias significativas respecto al control $(C)(P<0,050)$.

\subsection{Efecto de los acolchados sobre la disponibilidad de agua para las plantas}

En el conjunto de control (C), el claro efecto de la estacionalidad mediterránea sobre las precipitaciones se ha reflejado en el coeficiente de variación para el SWC, CV>80,0\% (Tabla 5). Dicha estacionalidad comprendería la alternancia de eventos de precipitación de intensidad elevada con períodos prolongados de sequía (Fig. 1) (Don et al., 2014; Hueso-González et al., 2015; Young et al., 2015). En términos generales, esto se relacionaría con el contenido volumétrico de agua presente en el perfil edáfico. Así, con independencia de la profundidad, nuestros resultados muestran que el SWC se encontró por debajo del punto de marchitez (WP) durante un período de 6 meses dentro del año hidrológico (Fig. 7). Por tanto, el agua contenida en el perfil no estaría disponible para ser utilizada por la vegetación, que se vería sometida a un fuerte estrés hídrico. Este hecho nos explica el elevado porcentaje de marras encontrado para los suelos no enmendados (Tabla 2) (Hasee y Rose, 1993; Whisenant et al., 1999; South, 2000; Hueso-González et al., 2015, 2016; Muñoz-Rojas et al., 2016c). 
Tabla 5. Humedad edáfica (\%) a 5 y $10 \mathrm{~cm}$ del perfil (Media anual, desviación estándar (SD), coeficiente de variación (CV). El periodo de estudio comprende desde octubre 2011 a marzo 2014. C: suelos reforestados y no enmendados; SM: acolchado de paja; PM: acolchado de astillas de pino carrasco (Pinus halepensis L.); * indica diferencias significativas respecto al control (C).

\begin{tabular}{|c|c|c|c|c|c|c|}
\hline & \multicolumn{3}{|c|}{$5 \mathrm{~cm}(\%)$} & \multicolumn{3}{|c|}{$10 \mathrm{~cm}(\%)$} \\
\hline & Media & $\mathrm{SD} \pm$ & $\mathrm{CV}(\%)$ & Media & $\mathrm{SD} \pm$ & $\mathrm{CV}(\%)$ \\
\hline C & 7,0 & 6,0 & 85,7 & 4,0 & 5,0 & 125,0 \\
\hline $\mathrm{SM}$ & 8,0 & 7,0 & 87,5 & 7,0 & 5,0 & 71,4 \\
\hline PM & 7,0 & 6,0 & 85,7 & 6,0 & 5,0 & 83,3 \\
\hline
\end{tabular}
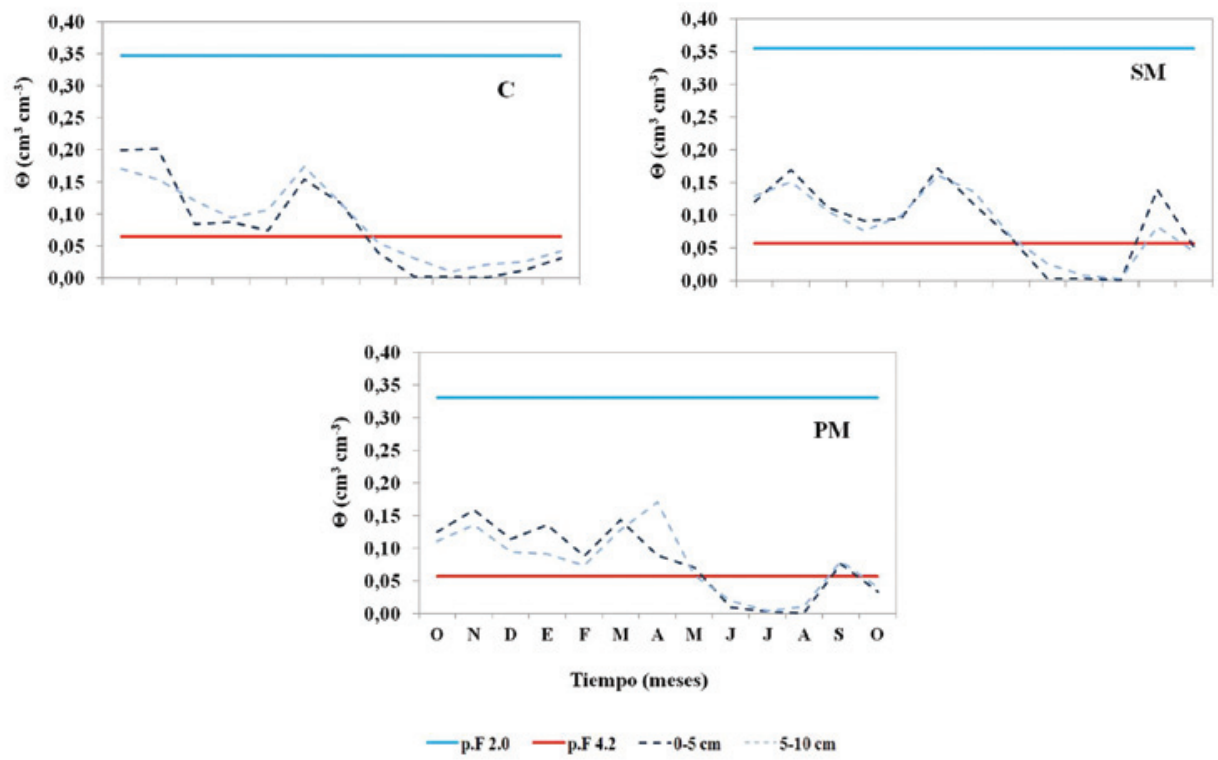

Figura 7. Evolución mensual del contenido de humedad del suelo en los diferentes manejos y su relación con la capacidad de retención hídrica a pF 2.0 (capacidad de campo) y p.F 4.2 (punto de marchitez permanente). $C$ : suelos reforestados y no enmendados; PM:acolchado de restos de poda de pino (Pinus halepensis Mill.); SM: acolchado de paja.

En otro sentido, Ros et al. (2006) y Hueso-González et al. (2015) han demostrado que la adición al suelo de acolchados orgánicos consigue incrementar la humedad media en el perfil edáfico ya que se minimizan los procesos de la evapotranspiración y se favorecen los procesos de infiltración. En esta línea, los tratamientos SM y PM han conseguido mantener los valores de SWC relativamente constantes en profundidad y los coeficientes de variación medidos (CV) han sido inferiores a los observados para el conjunto control (Tabla 5). Esto se interpreta como una mayor estabilidad en el SWC a lo largo del año hidrológico y consecuentemente, se relaciona con una mayor disponibilidad de agua para la vegetación reforestada, minimizando los efectos del verano mediterráneo. Así la Figura 7 muestra, 
para los tratamientos SM y PM, que el número de meses a lo largo del año hidrológico con agua disponible para la vegetación (AWC) se incrementa respecto al control y, en consecuencia, se consigue reducir el estrés hídrico post-plantación. Este aumento del AWC para los tratamientos SM y PM justificaría el incremento anteriormente descrito en las tasas de supervivencia para todas las especies (Tablas 2 y $4, P=0,00$ ).

En la misma línea, se relaciona el incremento registrado en el AWC con el incremento de las plantas en altura y diámetro (Figs. 2 y 3 ; Tabla $4 ; P=0,00 ; P=0,00 ; P=0,00$; $P=0,04$, respectivamente).

Finalmente en este estudio no se han podido establecer diferencias entre los tratamientos SM y PM. Esto es debido a que tanto las tasas de supervivencia como el crecimiento de las plantas han sido bastante similares (Tabla 1 y Figs. 2, 3, 4, 5 y 8).

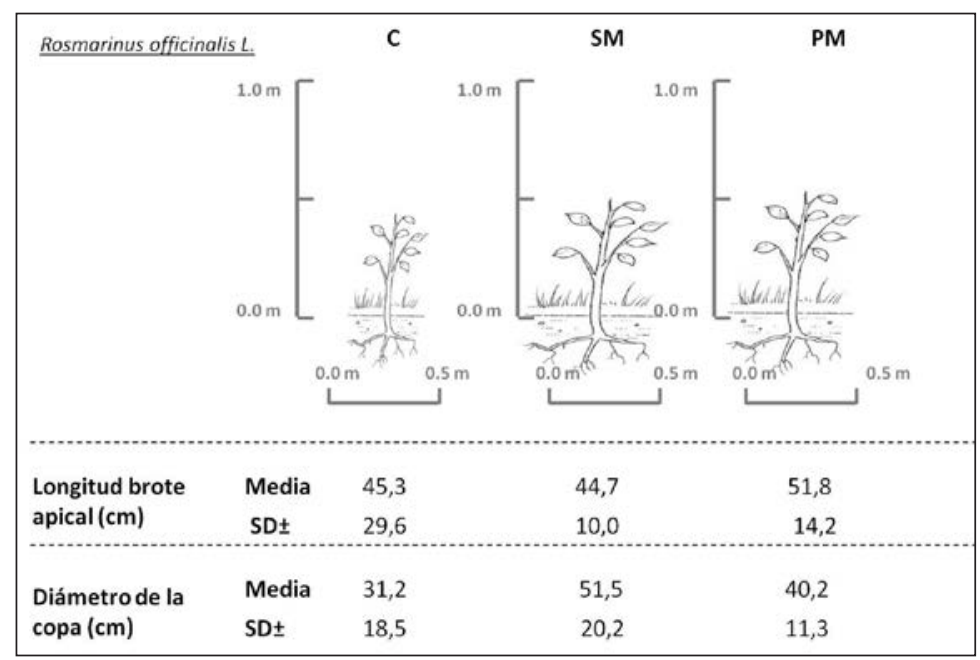

Figura 8. Rosmarinus officinalis L.: Crecimiento en altura de la vegetación (cm) y diámetro máximo de la copa 30 meses después de la reforestación. C: suelos reforestados y no enmendados; PM: acolchado de restos de poda de pino (Pinus halepensis Mill.); SM: acolchado de paja. * indica diferencias significativas respecto al control $(C)(P<0,050)$.

\section{Conclusiones}

En condiciones de clima seco-mediterráneo el uso de acolchados orgánicos como complemento a los proyectos de revegetación podría ayudar al establecimiento de los plantones. En este estudio, 30 meses después de la reforestación, no se observan diferencias significativas en el contenido de carbono orgánico del suelo para los acolchados de paja y poda respecto al control. Sin embargo, sí se han encontrado diferencias en la cantidad y disponibilidad de agua útil para la vegetación. Así, el uso de acolchados de paja y poda permite incrementar el contenido volumétrico de agua en el perfil edáfico y consecuentemente, aumentar la cantidad de agua disponible para las plantas. Esto se interpreta como una reducción del estrés hídrico post-transplante, que permitiría amorti- 
guar los efectos del verano mediterráneo. Todo ello generaría un efecto positivo para la supervivencia y crecimiento de las plantas reforestadas. Podemos concluir por tanto, que de cara a la gestión forestal, el tratamiento del suelo con acolchados orgánicos se traduce en un incremento del éxito de los programas de reforestación.

\section{Agradecimientos}

Este estudio ha sido financiado por el proyecto de investigación P09-RNM-5057 (Consejería de Innovación, Ciencia y Empleo de la Junta de Andalucía), así como por el Campus de Excelencia Internacional de Andalucía Tech. Finalmente, los autores quieren agradecer a la empresa TRAGSA su apoyo técnico durante la puesta en marcha de la red experimental.

\section{Referencias}

Almendros, G., González-Vila, J., González-Pérez, J.A., Knicker, H., de la Rosa, J.M. 2010. Protocolos y técnicas para evaluar el impacto del fuego sobre la materia orgánica. En A. Cerdà, A. Jordán (Eds.), Actualización en métodos y técnicas para el estudio de los suelos afectados por incendios forestales. Cátedra de Divulgación de la Ciencia, Valencia, pp. 291-319.

Benigno, S.M., Dixon, K.W., Stevens, J.C. 2013. Increasing soil water retention with native sourced mulch improves seedling establishment in postmine Mediterranean sandy soils. Restoration Ecology 21, 617-626. http://doi.org/10.1111/j.1526-100X.2012.00926.x.

Bochet, E., García-Fayos, P. 2004. Factors controlling vegetation establishment and water erosion on motorway slopes in Valencia, Spain. Restoration Ecology 12, 166-174. http://doi.org/10.1111/ j.1061-2971.2004.0325.x.

Bochet, E., García-Fayos, P., Alborch, B., Tormo, J. 2007. Soil water availability effects on seed germination account for species segregation in semiarid roadslopes. Plant Soil 295, 179-191. http://doi.org/10.1007/s11104-007-9274-9.

Bolker, B.M., Brooks, M.E., Clark, C.J., Geange, S.W., Poulsen, J.R., Stevens, M.H.H., Wite, J-S.S. 2009. Generalized linear mixed models: a practical guide for ecology and evolution. Trends in Ecology \& Evolution 24, 127-135. http://doi.org/10.1016/j.tree.2008.10.008.

Breton, V., Crosaz, Y., Rey, F. 2016. Effects of wood chip amendments on the revegetation performance of plant species on eroded marly terrains in a Mediterranean mountainous climate (Southern Alps, France). Solid Earth 7, 599-610. http://doi.org/10.5194/se-7-599-2016.

Brevik, E.C. 2009. Soil health and productivity. In Soils, plant growth and crop production. In W. Verheye (Ed.), Encyclopedia of Life Support Systems (EOLSS), UNESCO, París.

Caldwell, M.M. 1976. Root extension and water absorption. In O.L. Lange, L. Kappen, E.D. Schulze (Eds.), Water and plant life. Problems and modern approaches, Springer Verlang, Berlin, pp. 73-86.

Casals,P, Cortina J., Bottner,P., Coûteaux, M.M., Vallejo, V.R. 2000.CO2 efflux from a Mediterranean semi-arid forest soil. I. Seasonality and effects of stoniness. Biogeochemistry 48, 261-281. http://doi.org/10.1023/A:1006289905991.

Castillo, V.M., Martínez-Mena, M., Albadejo, J. 1997. Runoff and soil erosion response to vegetation removal in a semiarid environment. Soil Science Society of American Journal 61, 1116-1121. http://doi.org/10.2136/sssaj1997.03615995006100040018x.

Castillo, V., Querejeta, J., Albaladejo, J. 2001. Disponibilidad hídrica en repoblaciones de Pinus halepensis Mill. en medios semiáridos: efectos de los métodos de preparación del suelo, III Congreso Forestal Español, pp. 94-99. 
Castro, J., Zamora, R., Hodar, J.A., Gómez, J.M. 2002. Use of shrubs as nurse plants: a new technique for reforestation in Mediterranean mountains. Restoration Ecology 10, 297-305. http://doi. org/10.1046/j.1526-100X.2002.01022.x.

Cerdà, A. 2001. Effects of rock fragments cover on soil infiltration, inter-rill runoff and erosion. European Journal of Soil Science 52, 59-68. http://doi.org/10.1046/j.1365-2389.2001.00354.x.

Cerdà,A., González-Pelayo, O., Giménez-Morera,A., Jordán,A., Pereira, P., Novara, A, Brevik, E.C., Prosdocimi, M., Mahmoodfabadi, M., Keesstra, S., García Orenes, F., Ritsema, C. 2016. The use of barley straw residues to avoid high erosion and runoff rates on persimmon plantations in Eastern Spain under low frequency-high magnitude simulated rainfall events. Soil Research 54 (2), 154-165. http://doi.org/10.1071/SR15092.

Chaudhuri, S., McDonald, L.M., Skousen, J., Pena-Yewtukhiw, E.M. 2015. Soil organic carbon molecular properties: effects of time since reclamation in a minesoil chronosequence. Land Degradation and Development 26 (3), 237-248. http://doi.org/10.1002/ldr.2202.

Cohen-Fernandez, A.C., Naeth, M.A. 2013. Erosion control blankets, organic amendments and site variability influenced the initial plant community at limestone quarry in the Canadian Rocky Mountains. Biogeosciences 10, 5243-5253. http://doi.org/10.5194/bg-10-5243-2013.

COM. 2002. Towards a thematic strategy for soil production. Communication for the Commission to the Council, the European Parliament, the Economic and Social Committee and the Committee of the Regions. COM (2002). 179 end. Brussels, 16.4.2002.

Donn, S., Wheatley, R.E., Mckenzie, B.M., Loades, K.M., Hallett, P.D. 2014. Improved soil fertility from compost amendments increases root growth and reinforcement of surface soil on slope. Ecological Engineering 71, 458-465. http://doi.org/10.1016/j.ecoleng.2014.07.066.

Duryea, M.L., Jeffery, E.R., Hermasen, A.L. 1999. Will subterranean termites consume landscape mulches? Journal of Arboriculture 25, 88-97.

Eaton, J.M., McGoff, N.M., Byrne, K.A., Leahy, P., Kiely, G . 2008. Land cover change and soil organic C stocks in the Republic of Ireland 1851-2000. Climatic Change 91, 317-334, 2008. http://doi.org/10.1007/s10584-008-9412-2.

Eldridge, J.D., Redente, E.F., Paschke, M. 2012. The use of seedbed modifications and wood chips to accelerate restoration of well pad sites in western Colorado, USA. Restoration Ecology 20, 524-531. http://doi.org/10.1111/j.1526-100X.2011.00783.x.

FAO. 2006. World reference base for soil resource. A framework for a international classification, correlation and communication. FAO, Roma, 145.

Ferreras, L., Gómez, E., Torresani, S., Firpo, I., Rotondo, R. 2006. Effect of organic amendments on some physical, chemical and biological properties in a horticultural soil. Bioresource Technology 97 (4), 635-640. http://doi.org/10.1016/j.biortech.2005.03.018.

Fons, J., Vallejo, V.R. 1997. Humus form patterns in some Mediterranean forests. Ann. Sci. For. 55, 493-499.

Franco-Otero, V.C., Soler-Rovira, P., Hernández, D., López-De-Sá, E., Plaza, C.S. 2011. Short-term effects of organic municipal wastes on wheat yield, microbial biomass, microbial activity, and chemical properties of soil. Biology and Fertility of Soils 12 (2), 1257-1268. http://doi. org/10.1007/s00374-011-0620-y.

García-Gómez, A., Bernal-Calderón, M.P., Roig, A. 2005. Humification parameters, organic waste management, organic matter. Compost Science and Utilization 13 (2), 127-135.

García-Orenes, F., Roldán, A., Mataix-Solera, J., Cerdà, A., Campoy, M., Arcenegui, V., Caravaca, F. 2012. Soil structural stability and erosion rates influenced by agricultural management practices in a semi-arid Mediterranean agro-ecosystem. Soil Use and Management 28, 571-579. http:// doi.org/10.1111/j.1475-2743.2012.00451.x. 
Gómez, M.J., Baladanis, P., Peter, A. (Eds.) 1999. Example of desertification mitigation: integral forest fire prevention in Comunidad Valenciana (Spain). Research results and policity implications. Office for Official Publications of the European Communities. Luxembourg.

Gómez-Aparicio, L., Zamora, R., Gómez, J.M., Hódar, Castro, J., Baraza., E. 2004. Applying plant facilitation to forest restoration: A meta-analysis of the use of shrubs as nursery plants. Ecological Applications 14 (4), 1128-1138.

González-Ubierna, S., Jorge-Mardomingo, I., Carrero-González, B., De la Cruz, M.T., Casermeiro, M.A. 2012. Soil organic matter evolution after the application of high doses of organic amendments in a Mediterranean calcareous soil. Soils and Sediments, 12 (8) 1257-1268. http:// doi.org/10.1007/s11368-012-0516-y.

Grossnickle, S.C. 2000. Ecophysiology of northern spruce species. The performance of planted seedlings. NCR Research Press, Ottawa, Ontario, Canada, 409.

Guerrero, C., Gómez, I., Moral, R., Mataix-Solera, J., Mataix-Beneyto, J., Hernández, T. 2001. Reclamation of a burned forest soil with municipal waste compost: macronutrient dynamic and improved vegetation cover recovery. Bioresource Technology 76, 221-227. http://doi. org/10.1016/S0960-8524(00)00125-5.

Hasse, D.L., Rose, R. 1993. Soil moisture stress induces transplant shock in stored and unstored $2+0$ Douglas-Fir seedlings of varying root volumes. Forest Science 39, 275-294.

Hosseini Bai, S., Blumfield, T.J., Reverchorn, F. 2014. The impact of mulch type on soil carbon and nitrogen pools in a sloping site. Biology and Fertility of Soils 50,37-44. http://doi.org/10.1007/ s00374-013-0829-z.

Hueso-González, P. 2014. Estrategias para conservación del suelo y recuperación de la cubierta vegetal en un ambiente ecotono mediterráneo. Tesis doctoral. Servicio de Publicaciones y Divulgación Científica de la Universidad de Málaga, pp. 15-38. http://hdl.handle. net/10630/9548.

Hueso-González,P., Martínez-Murillo, J.F., Ruiz Sinoga,J.D. 2014. The impact of organic amendments on forest soil properties under Mediterranean climatic conditions. Land Degradation \& Development 25, 604-612. http://doi.org/10.1002/ldr.2296.

Hueso-González, P., Martínez-Murillo, J.F., Ruiz-Sinoga, J.D. 2016. Effects of topsoil treatments on afforestation in a dry-Mediterranean climate (Southern Spain). Solid Earth 7, 1479-1489. http:// doi.org/10.5194/se-2016-98.

Hueso-González, P., Ruiz-Sinoga, J.D., Martínez-Murillo, J.F., Lavee, H. 2015. Overland flow generation mechanisms affected by topsoil treatment: application to soil conservation. Geomorphology 228, 796-804. http://doi.org/10.1016/j.geomorph.2014.10.033.

IBM Corp. Released. 2012. IBM SPSS Statistics for Windows, Version 21 .0. IBM Corp., Armonk, NY.

Jensen, H.L. 2009. The microbiology of farmyard manure decomposition in soil II, Decomposition of cellulose. Journal of Agricultural Science 21, 81-100. http://doi.org/10.1017/S0021859600007966.

Jiménez, M.N., Fernández-Ondoño, E., Ripoll, M.A., Castro-Rodríguez, J., Huntsinger, L., Bruno Navarro, F. 2013. Stones and organic mulches improve the Quercus Ilex L. afforestation success under Mediterranean climatic conditions. Land Degradation \& Development 27, 357-365. http://doi.org/10.1002/ldr.2250.

Jordán, A., Zavala, L.M., Gil, J. 2010. Effects of mulching on soil physical properties and runoff under semi-arid conditions in southern Spain. Catena 81, 77-85. http://doi.org/10.1016/j. catena.2010.01.007.

Jordán, A., Zavala, L.M., Muñoz-Rojas, M. 2011. Mulching, effects on soil physical properties. In J. Glinski, J. Horabik, J. Lipiec (Eds.), Encyclopedia of Agrophysics. Springer, Berlin, pp. 492-496.

Lal R. 1997. Residue management, conservation tillage and soil restoration for mitigating greenhouse effect by CO2-enrichment. Soil and Tillage Research 43, 87-107. http://doi.org/10.1016/S01671987(97)00036-6. 
Le Houérou,H.N. 2010. Restoration and rehabilitation of Arid and Semiarid Mediterranean ecosystems in North Africa and West Asia, A review, Arid Soil Research and Rehabilitation 14, 3-14. http:// doi.org/10.1080/089030600263139.

LUCDEME. 1986. Proyecto LUCDEME. Mapa de suelos 1:100.000. Motril-1055. Ministerio de Agricultura, Pesca y Alimentación, ICONA. Universidad de Granada, Facultad de Ciencias, Departamento de Edafología.

Maestre, F.T., Cortina, J. 2002. Spatial patterns of surface soil properties and vegetation in a Mediterranean semi-arid steppe. Plant and Soil 241, 279-291. http://doi. org/10.1023/A:1016172308462.

Maestre, F.T., Cortina, J., Bautista, S., Bellot, J., Vallejo, V.R. 2003. Small-scale environmental heterogeneity and spatial-temporal dynamics of seedling establishment in a semiarid degraded ecosystem. Ecosystems 6, 630-643. http://doi.org/10.1007/s10021-002-0222-5.

Maestre,F.T., Huesca, M.,Zaady,E., Bautista, S., Cortina, J. 2002. Infiltration, penetration resistance and microphytic crust composition in contrasted microsites within a Mediterranean semi-arid steppe. Soil Biology and Biochemistry 34, 895-898. http://doi.org/10.1016/S0038-0717(02)00021-4.

Martínez-Fernández, J., Lopez-Bermúdez, F., Martínez-Fernández, J., Romero-Díaz, M.A.1995. Land use and soil vegetation relationships in Mediterranean ecosystem: El Ardal, Murcia, Spain. Catena 25, 153-167. http://doi.org/10.1016/0341-8162(95)00007-F.

Martínez-Murillo, J.F., Hueso-González, P., Ruiz-Sinoga, J.D., Lavee, H. 2016a. Short-term experimental fire effects in soil and water losses in southern of Spain. Land Degradation \& Development 27, 1513-1522. http://doi.org/10.1002/ldr.2504.

Martínez-Murillo, J.F., Remond, R., Hueso-González, P., Ruiz-Sinoga., J.D. 2016b. Vegetation cover resilence in a burned montainous area: the Rio Verde Wildfire (Province of Málaga, South of Spain). Pirineos, Revista de Ecología de Montaña 171, 1-15. http://doi.org/10.3989/ pirineos.2016.171008.

Ministerio de Medio Ambiente. 2002a. Plan Forestal Español. Documento de debate. http://www. mma.es/conserv_nat/planes/planifor/pfeindex.html.

Ministerio de Medio Ambiente. 2002b. Plan Forestal Español. Documento de debate. Anexos. http:// www.mma.es/conserv_nat/planes/planifor/pfeindex.html.

Montgomery, D.R. 2007. Soil erosion and agricultural sustainability. Proceedings of the National Academy of Sciences of the United States of America 104, 13268-13272.

Muñoz-Rojas, M., Erickson, T. E., Dixon, K. W., Merritt, D. 2016a. Soil quality indicators to assess functionality of restored soils in degraded semiarid ecosystems. Restoration Ecology 24: S43-S52. http://doi.org/10.1111/rec.12368.

Muñoz-Rojas, M., Erickson,T.E., Martini, D., Dixon, K.W., Merritt,D.J.2016b. Soil physicochemical and microbiological indicators of short, medium and long term post-fire recovery in semi-arid ecosystems. Ecological Indicators 63, 14-22. http://doi.org/10.1016/j.ecolind.2015.11.038.

Muñoz-Rojas, M., Erickson, T.E., Martini, D., Dixon, K.W., Merritt, D.J. 2016c. Climate and soil factors influencing seedling recruitment of plant species used for drylands restoration. SOIL 2, 287-298. http://doi.org/10.5194/soil-2-287-2016.

Novara, A, Gristina, L, Bodì, MB, Cerdà, A. 2011.The impact of fire on redistribution of soil organic matter on a mediterranean hillslope under maquia vegetation type. Land Degradation \& Development 22 (6), 530-536. http://doi.org/10.1002/ldr.1027.

Nykvist, N. 1983. Soil erosion, The scientific Magazine of Swedish Forestry Association, $N^{\circ} 1 / 83,48$. Ojeda, G., Alcañiz, J.M., Ortiz, O. 2003. Runoff and losses by erosion in soils amended with sewage sludge. Land Degradation \& Development 14 (6), 563-573. http://doi.org/10.1002/ldr.580.

Prats, S.A., Malvar, M.C., Vieira, D.C.S., MacDonald, L., Keizer, J.J. 2013. Effectiveness of hydromulching to reduce runoff and erosion in a recently burnt pine plantation in central Portugal. Land Degradation \& Development 1319-1333. http://doi.org/10.1002/ldr.2236. 
Prosdocimi, M., Jordán, A., Tarolli, P., Keesstra, S., Novara, A., Cerdà, A. 2016. The immediate effectiveness of barley straw mulch in reducing soil erodibility and surface runoff generation in Mediterranean vineyards. Science of the Total Environment 547, 323-330. http://doi. org/10.1016/j.scitotenv.2015.12.076.

Richards, L.A. 1947. Pressure membrane apparatus: construction and use. Agricultural Engineering 28, 451-454.

Rojo, L., Enne, G., Zanolla, Ch., Peter, D. (Eds.) 2000. Managing desertification in a national context. Desertification in Europe: mitigation strategies, land use planning. Officine for Official Publications of the European Communities, Luxembourg.

Ros, M., Pascual, J.A., García, C., Hernández, T., Insam, H. 2006. Hydrolase activities, microbial biomass and bacterial community in a soil after long-term amendment with different composts. Soil Biology and Biochemistry 38 (12),3443-3452.http://doi.org/10.1016/j.soilbio.2006.05.017.

Shachak, M., Sachs, M., Moshe, I. 1998. Ecosystem management of deserted scrubslands in Israel. Ecosystem 1, 475-483.

Shazana, M.A.R.S., Shamshuddin, J., Fauziah, C.I., Syed Omar, S.R. 2013. Alleviating the infertility of an acid sulphate soil by using ground basalt with or without lime and organic fertilizer under submerged conditions. Land Degradation \& Development 24 (2), 129-140. http://doi. org/10.1002/ldr.1111.

South, D.B. 2000. Planting morphologically improved pine seedlings to increase survival and growth. Forestry and Wildlife Research Series, no. 1, Alabama Agricultural Experiment Station, Auburn University, Alabama, 12 pp.

Srinivasarao, C.H., Venkateswarlu, B., Lal, R., Singh, A.K., Kundu, S., Vittal, K.P.R., Patel, J.J., Patel, M.M. 2013. Long-term manuring and fertilizer effects on depletion of soil organic carbon stocks under pearl millet-cluster bean-castor rotation in Western India. Land Degradation \& Development 25 (2), 173-183. http://doi.org/10.1002/ldr.1158.

Stakman, W.P., Valk, G.A., Van der Harst, G.G. 1969. Determination of soil moisture retention curves. I. Sand-box apparatus. Range $\mathrm{pF} 0$ to 2.7. I.C.W., 19 pp.

Tejada, M., González, J.L. 2013. Effects of the application of a compost originating from crushed cotton gin residues on wheat yield under dryland conditions. European Journal of Agronomy 19, 357-368. http://doi.org/10.1016/S1161-0301(02)00089-8.

Thornes, J.B. 1985. The ecology of erosion. Geography, 70 (3), 222-235.

Thornes, J.B. 1990. The interaction of erosional and vegetation dynamics in land degradation: spatial outcomes. In J.B. Thornes (Eds.) Vegetation and Erosion, Wiley, New York, pp. 41-54.

Tongway, D.J., Ludwing, J.A. 1996. Rehabilitation of Semiarid Landscape in Australia, I, Restoring productive soil patches. Restoration Ecology 4, 388-397. http://doi.org/10.1111/j.1526100X.1996.tb00191.x.

Vallejo, V.R., Bautista, S., Cortina, J. 2000. Restoration for soil protection after disturbance. Life and Ecosystem in the Mediterranean, Trabaud, L. (Eds.), WIT Press, Southampton, United Kingdom.

Van Wesemael B., Veer, M.A.C.. 1992. Soil organic matter accumulation, litter decomposition and humus forms under mediterranean-type forests in southern Tuscany, Italy. European Journal of Soil Science 43, 133-144. http://doi.org/10.1111/j.1365-2389.1992.tb00125.x.

Whisenant, S.G. 1999. Repairing Damage Windlands. Cambridge University Press, Cambridge, United Kingdom.

Woods, S.R., Fehmi, J.S., Backer, D.M. 2012. An assessment of revegetation treatments following removal of invasive Pennisetum ciliare (buffelgrass). Journal of Arid Environment 87, 168175. http://doi.org/10.1016/j.jaridenv.2012.06.009.

Young, I., Renault, S., Markham, J. 2015. Low levels of organic amendments improves fertility and plant cover on non-acid generating gold mine tailings. Ecological Engineering 74, 250-257. http://doi.org/10.1016/j.ecoleng.2014.10.026. 